\title{
Automatic Freeway Incident Detection for Free Flow Conditions: A Vehicle Reidentification Based Approach Using Image Data from Sparsely Distributed Video Cameras
}

\author{
Jiankai Wang ${ }^{1,2}$ and Agachai Sumalee ${ }^{1,3}$ \\ ${ }^{1}$ Department of Civil and Environmental Engineering, The Hong Kong Polytechnic University, Hung Hom, Kowloon, Hong Kong \\ ${ }^{2}$ Central Research Institute (CRI), Huawei Technologies Co., Ltd., Shenzhen 518129, China \\ ${ }^{3}$ Department of Civil Engineering, Faculty of Engineering, King Mongkut's Institute of Technology Ladkrabang, \\ Bangkok 10520, Thailand
}

Correspondence should be addressed to Agachai Sumalee; ceasumal@polyu.edu.hk

Received 14 December 2014; Revised 29 April 2015; Accepted 1 May 2015

Academic Editor: Erik Cuevas

Copyright $\odot 2015$ J. Wang and A. Sumalee. This is an open access article distributed under the Creative Commons Attribution License, which permits unrestricted use, distribution, and reproduction in any medium, provided the original work is properly cited.

\begin{abstract}
This paper proposes a vehicle reidentification (VRI) based automatic incident algorithm (AID) for freeway system under free flow condition. An enhanced vehicle feature matching technique is adopted in the VRI component of the proposed system. In this study, arrival time interval, which is estimated based on the historical database, is introduced into the VRI component to improve the matching accuracy and reduce the incident detection time. Also, a screening method, which is based on the ratios of the matching probabilities, is introduced to the VRI component to further reduce false alarm rate. The proposed AID algorithm is tested on a $3.6 \mathrm{~km}$ segment of a closed freeway system in Bangkok, Thailand. The results show that in terms of incident detection time, the proposed AID algorithm outperforms the traditional vehicle count approach.
\end{abstract}

\section{Introduction and Literature Review}

Traffic incidents have been widely recognized as a serious problem for its negative effects on traffic congestion and safety [1]. Under heavy traffic condition, one minor incident could result in gridlock and hence serious traffic congestion. In addition, traffic injuries are likely to be more severe if incidents occur at higher speeds (e.g., under free flow condition). Statistics also suggest the high chance of a more sever secondary accident following the initial incident on freeway $[2,3]$. An ability to detect incident in a timely and accurate manner would allow the traffic manager to efficiently remove the incident, to notify the follow-up traffic of the incident, and to better manage the traffic for minimizing the negative impact caused by the incident. Therefore, considerable research efforts have been dedicated to the development of automatic incident detection (AID) algorithms by utilizing the traditional detectors (i.e., inductive loops) over the past few decades (e.g., the California algorithm series [4] and
McMaster algorithm [5]). The underlying assumption of these algorithms is that the aggregated traffic parameters (e.g., travel time and traffic flow) would change dramatically when incidents occur under congested situation. By comparing the real-time traffic data with the incident-free data, one can determine the likelihood that an incident has happened. Based on the above-mentioned principle, various advanced data mining approaches (e.g., neural network [6], Bayesian network $[7,8]$, and principal component analysis [9]) were adopted for detecting the abnormal traffic delay or abrupt change in traffic flow pattern. However, most of the existing incident detection algorithms are specifically designed for congested traffic conditions and may not be applicable for free flow situations.

Under free flow condition, a drop in traffic capacity due to a traffic incident (e.g., one lane blocking) may not cause any traffic delay or substantial change in traffic flow pattern. Therefore, it is no longer feasible to detect the incident through analyzing the aggregated traffic parameters. 
To handle the aforementioned challenge, research attention has shifted away from the data mining approach based on macroscopic traffic data, towards considering the continuous tracking of individual vehicle by using the microscopic vehicle data (e.g., vehicle trajectory and individual vehicle feature). The rationale behind this idea is straightforward. For a closed freeway system, if one can track all the vehicles along the designated points, a disappearance of any vehicle movement (or nonmoving vehicle) between consecutive points can be classified as a potential incident. Based on this principle, various emerging technologies, such as GPS technology [10] and cellular phones [11], has been utilized for collecting the Lagrangian measurements (i.e., individual vehicle trajectory), which eventually could be used for identifying the "incident" vehicle. Despite their theoretical simplicity, the successes of these approaches heavily rely on the high level of market penetration (in principle, 100\% of penetration rate is required) of the in-vehicle equipment (e.g., GPS device) and the driver's willingness to provide the location information. To compensate for this, Shehata et al. [12] conducted a study to detect the nonmoving vehicle (i.e., caused by incident) from the fixed video camera (i.e., Eulerian measurement) by using image processing techniques. Although this method appears to be theoretically sound, the deployment of such system requires the installations of cameras at all key locations along the freeway, which may not be feasible due to the limitation of public resources. In practice, the fixed traffic sensors (e.g., loop detectors and video cameras) cannot cover the entire freeway system and are generally sparsely distributed [13]. In this regard, Fambro and Ritch [14] conducted a pioneering study to trace and identify the "missing" vehicle through analyzing the vehicle count data obtained at two consecutive loop detectors, which is also referred to as the vehicle count approach. Given the vehicle speed at upstream, the arrival time window at downstream could be estimated. By comparing the vehicle counts in this arrival time window with the corresponding vehicle counts at upstream, one may be able to identify the missing vehicle (if any) for incident detection. However, it is also noteworthy that the overlapping of arrival time windows of different vehicles would lead to a significant increase in the detection time (this will be discussed in more detail in Section 3.1). To further reduce the incident detection time, much attention has been paid to track the vehicle by utilizing the emerging automatic vehicle identification (AVI) systems: automatic number plate recognition [15] or Bluetooth identification technology [16]. Although the AVI technologies enable a more efficient tracking of vehicles across consecutive points by accurately matching their unique identity (e.g., plate number and media access control address), privacy concerns may arise as the consequence of this matching process of vehicle identity. In this case, the vehicle reidentification (VRI) scheme, which does not intrude driver's privacy, provides a tool to devise a more practical and effective incident detection algorithm under free flow condition.

Generally, vehicle reidentification (VRI) is a process of matching nonunique vehicle signatures (e.g., waveform [17], vehicle length [18], and vehicle color $[19,20])$ from one detector to the next one in the traffic network. On one hand, the nonuniqueness of the vehicle signature would allow the VRI system to track the vehicle anonymously at two consecutive detectors [21] and, hence, identify the "missing" vehicle due to an incident. On the other hand, this property of very nonuniqueness imposes a great challenge on the development of the vehicle signature matching method. To improve the matching accuracy, Coifman [22] proposed a vehicle platoon matching method such that the lengths of vehicle platoons were compared. To further consider the noise and uncertainty of vehicle signature, Kwong et al. [23] introduced a statistical matching method in which the vehicle signature is treated as a random variable, and a probabilistic measure is calculated for matching decision making. During the past few years, the authors also developed a VRI system $[24,25]$ by utilizing the emerging video image processing systems [26]. Various detailed vehicle features (e.g., vehicle color, length, and type) were extracted and a probabilistic data fusion rule was then introduced to combine these features to generate a matching probability (i.e., posterior probability) for reidentification purpose. To account for the large variance in travel time under dynamic traffic condition, the proposed VRI system also introduced a prior (fixed) time window constraint, which sets the upper and lower bounds of the vehicle travel time, to rule out the unlikely candidate vehicles. However, it is noteworthy that the aforementioned VRI systems were specifically designed for the purpose of traffic data collection (e.g., travel time). To our knowledge, very few studies were explicitly conducted to investigate the potential feasibility of utilizing VRI system for incident detection. Also, as the existing VRI systems cannot guarantee an accurate matching due to the nonuniqueness of the vehicle signatures, the mismatches between the upstream and downstream vehicles may potentially lead to false alarms when they were applied for incident detection. To sum up, the current VRI systems are not readily transferable to the field of incident detection.

To this end, this paper aims to propose a VRI-based automatic incident detection algorithm under free flow condition. The revised VRI system is based on authors' previous work [24] with several major changes to cope with the purpose of incident detection, which eventually give rise to the incidentdetection-oriented VRI.

(i) Note that in the work of [24] a unified and fixed time window constraint is imposed on all the vehicles. However, the vehicles would maintain a relatively stable speed under free flow condition, which allows for the estimation of a flexible time window for each individual vehicle. Therefore, this incident-detectionoriented VRI would introduce a flexible time window to further improve the matching accuracy and reduce the incident detection time.

(ii) Rather than finding the matching results between two sets of vehicles (i.e., upstream and downstream sets of vehicles), the incident-detection-oriented VRI attempts to make an instant matching decision for each individual vehicle such that the "missing" vehicle can be identified promptly. In this study, the statistical model regarding the vehicle feature is built up, and the 
matching probability for each pair of vehicles (i.e., the posterior probability of each potential match based on the observation of the vehicle signatures) is explicitly calculated.

(iii) Last but not least, a screening method (i.e., thresholding process), which is based on the ratios of the matching probabilities, is introduced to screen out the mismatched vehicles for reducing the false alarm rate.

The rest of the chapter is organized as follows. Section 2 describes the traffic dataset collected for the algorithm development and evaluation. Mathematical notations are also provided in this section. In Section 3, the overall framework of the proposed automatic incident detection system is introduced. The description and analysis of the incidentdetection-oriented VRI system under free flow condition are proposed in the following two sections (Sections 4 and 5). In Section 6, simulated tests and real-world case studies are carried out to evaluate the performance of the proposed AID system against the traditional vehicle count approach. Finally, we close this chapter with the conclusion and future works.

\section{Dataset for Algorithm Development and Evaluation}

The test site is a $3.6 \mathrm{~km}$ long section of the closed threelane freeway in Bangkok, Thailand (i.e., the green section in Figure 1). At each station (i.e., location $10 \mathrm{~B}$ and $08 \mathrm{~B}$ in Figure 1) a gantry-mounted video camera, which is viewed in the upstream direction, is installed and two hours of video record (10 a.m. and noon on March 15, 2011) was collected. The frame rate of the video record is 25 FPS and the still image size is $563 \times 764$. As the detailed traffic data (especially the individual vehicle data) are not readily obtainable from the raw video record, the video image processing systems (VIPs) are then employed for extracting the required information (e.g., vehicle feature data and spot speed). In general, VIPs involve two major steps: vehicle detection and feature extraction. The first step is to digitize and store the raw video record for the detection subsystem to detect and capture the moving vehicle. The still image regarding the individual vehicle is stored for further application. In the second step, various image processing techniques are performed on the vehicle images to obtain the intrinsic feature data (e.g., color, length, and type). In the following, a brief review on the VIPs and associated image processing techniques are presented.

\subsection{Video Image Processing Systems}

2.1.1. Vehicle Detection. The success of vehicle detection largely depends on the degree that the moving object (vehicle) can be distinguished from its surroundings (background). In light of this, background estimation technology is employed in the detection subsystem. By calculating the media of a sequence of video frames, the background of the video image is obtained. Then, image segmentation technique is performed to identify the foreground object (vehicle). The still image including the detected vehicle is then clipped and stored for further feature extraction. Along with the detection of the vehicle, the associated arrival time $t$ and the spot speed $v$ are also collected. The normalized height of the vehicle image is adopted for representing the vehicle length $L$.

2.1.2. Vehicle Color Recognition. Color is one the most essential features for characterizing a vehicle. To reduce the negative effect of illumination changes, this paper has adopted the HSV (hue-saturation-value) color space to represent the vehicle image. Vehicle color recognition illustrated in this paper is conducted in two steps. First, the general RGB color images are converted into HSV color model-based images. Hue and Saturation values are then exploited for color detection, whereas $V$ (value) information is separated out from the color space. Second, a two-dimensional color histogram $C$ is formed to represent the distribution (frequency) of colors across a vehicle image. To be more specific, the hue and saturation channels are divided into 36 and 10 bins, respectively. Thus, a color feature vector $C$ with 360 elements is obtained. Each element of the feature vector is calculated as

$$
C_{i}=\frac{N_{i}}{N}, \quad 1 \leq i \leq 360,
$$

where $N_{i}$ is the number of pixels whose values fall within the bin $i$ and $N$ is the total number of pixels in the image.

2.1.3. Vehicle Type Recognition. Vehicle type feature provides the other important information to describe a vehicle. The template matching method [27] is utilized to recognize vehicle type. This method uses $L_{2}$ distance metric to measure the similarity between vehicle image and template images. Specifically, vehicles are classified into 6 categories. For each category, the corresponding template image $(T)$ is built. Finally, the normalized similarity value between the vehicle image $(I)$ and the $k$ th template image $(T)$ is given by

$$
S(k)=\frac{\sum_{m=1}^{\mathscr{M}} \sum_{n=1}^{\mathscr{N}}|I(m, n)-T(m, n)|^{2}}{\mathscr{G} \mathscr{M} \mathcal{N}},
$$

where $\mathscr{G}$ denotes the maximum gray level (255); $\mathscr{M}$ and $\mathcal{N}$ are the dimensions of the vehicle image. Thus, the vehicle type/shape feature $S$ is a vector that consists of the similarity score for each template. Detailed implementation of the VIP systems to traffic data extraction can be found in [24]. A formal description of the dataset obtained from the video record is presented in the following subsection.

2.2. Dataset Description and Notation. VIPs provide a large amount of traffic data to develop and validate the automatic incident detection algorithms. Let $U=\{1,2, \ldots, N\}$ denote the $N$ vehicles detected at upstream station during the time interval. $D=\{1,2, \ldots, M\}$ is the set of downstream vehicles. In addition, $t_{i}^{U}$ and $v_{i}^{U}$ are the associated arrival time and the spot speed of the $i$ th upstream vehicle, respectively. Accordingly, $t_{i}^{D}$ and $v_{i}^{D}$ are the corresponding arrival time and spot speed of the $i$ th downstream vehicle. As discussed above, for each detected individual vehicle, the intrinsic feature data (e.g., color, size, and length) are also obtained. Let $X_{i}^{U}=$ $\left\{C_{i}^{U}, S_{i}^{U}, L_{i}^{U}\right\}$ denote the signature of the $i$ th upstream vehicle, 


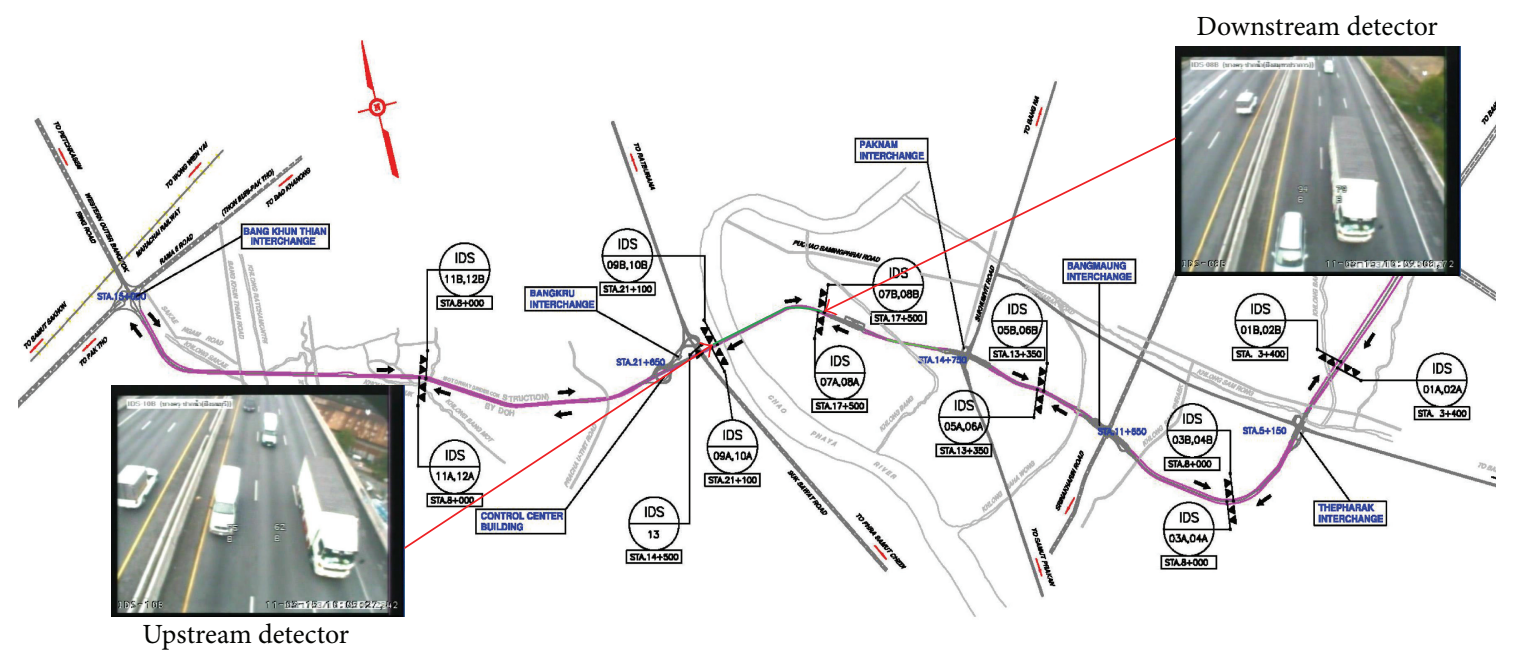

Figure 1: Test site in Bangkok, Thailand.

where $C_{i}^{U}$ and $S_{i}^{U}$ are the normalized color feature vector and type (shape) feature vector, respectively. $L_{i}^{U}$ denotes the normalized the length of vehicle $i$. Similarly, $X_{j}^{D}=\left\{C_{j}^{D}, S_{j}^{D}, L_{j}^{D}\right\}$ is the signature of the $j$ th downstream vehicle. To sum up, dataset from the VIPs during a time interval consists of the upstream vehicle dataset $\left\{\left(t_{i}^{U}, v_{i}^{U}, X_{i}^{U}\right), i=1,2, \ldots, N\right\}$ and the downstream vehicle set $\left\{\left(t_{j}^{D}, v_{j}^{D}, X_{j}^{D}\right), j=1,2, \ldots, M\right\}$. In order to quantify the difference between each pair of upstream and downstream vehicle signatures, several distance measures are then incorporated. Specifically, for a pair of signatures $\left(X_{i}^{U}, X_{j}^{D}\right)$, the Bhattacharyya distance [28] is utilized to calculate the degree of similarity between color features:

$$
d_{\text {color }}(i, j)=\left[1-\sum_{k=1}^{360} \sqrt{C_{i}^{U}(k) \cdot C_{j}^{D}(k)}\right]^{1 / 2},
$$

where $k$ denoted the $k$ th component of the color feature vector. The $L_{1}$ distance measure is introduced to represent the difference between the type feature vectors:

$$
d_{\text {type }}(i, j)=\sum_{k=1}^{q}\left|S_{i}^{U}(k)-S_{j}^{D}(k)\right|
$$

where $q$ is the number of vehicle type template and is taken as 6 in this study. The length difference is given by

$$
d_{\text {length }}(i, j)=\left|L_{i}^{U}-L_{j}^{D}\right| \text {. }
$$

Based on the video record collected at the test site, 3,628 vehicles are detected at both stations (10B and $08 \mathrm{~B})$ during the two-hour video record. For the purpose of the algorithm development and evaluation, these 3,628 pairs of vehicles are manually matched (i.e., reidentified) by the human operators viewing the video record frame by frame. In other words, the ground-truth matching results of the 3,628 pairs of vehicles are obtained in advance. The mean travel time is
170.9 seconds. The first 800 pairs of vehicle data are used for the model training and calibration (which are discussed in the following sections), while the rest of the vehicle dataset are used for the simulation test of the proposed automatic incident detection algorithm.

\section{Overall Framework of Automatic Incident Detection System}

The basic idea of incident detection under free flow condition is to track the individual vehicle so as to identify the missing vehicle due to an incident. Owning to its computational and theoretical simplicity, the vehicle count approach [14] is the most well-known free-flow incident detection algorithm. Thus, it is necessary to revisit this method in detail.

3.1. Vehicle Count Approach. The basic operation of the vehicle count approach is illustrated in Figure 2. When a vehicle $U_{i}$ arrives at upstream station at time $t_{i}^{U}$, the expected arrival time window $\left[t_{i}^{U}+L b_{i}, t_{i}^{U}+U b_{i}\right]$ of this vehicle at downstream station is estimated, where $L b_{i}$ and $U b_{i}$, respectively, represent the lower and upper bounds of the vehicle's travel time. If another vehicle $U_{j}$ is detected at upstream station, the corresponding arrival time window $\left[t_{j}^{U}+L b_{j}, t_{j}^{U}+U b_{j}\right]$ can also be obtained. Unsurprisingly, there may be overlap between these two time windows, and both of these two vehicles are likely to arrive at downstream during time interval $\left[t_{j}^{U}+L b_{j}, t_{i}^{U}+U b_{i}\right]$. The incident would then be detected by comparing the collected vehicle count data to the expected number of vehicles in the time interval. In the case that vehicle $U_{i}$ is missing, if vehicle $U_{j}$ arrives at downstream during time interval $\left[t_{j}^{U}+L b_{j}, t_{i}^{U}+U b_{i}\right]$, then the incident alarm will not be triggered until time $t_{j}^{U}+U b_{j}$, which is clearly later than the upper bound of the arrival time of vehicle $U_{i}$ (i.e., $t_{i}^{U}+U b_{i}$ ). Because of the overlapping between the time windows, the vehicle count approach, which is solely based on comparing the vehicle 


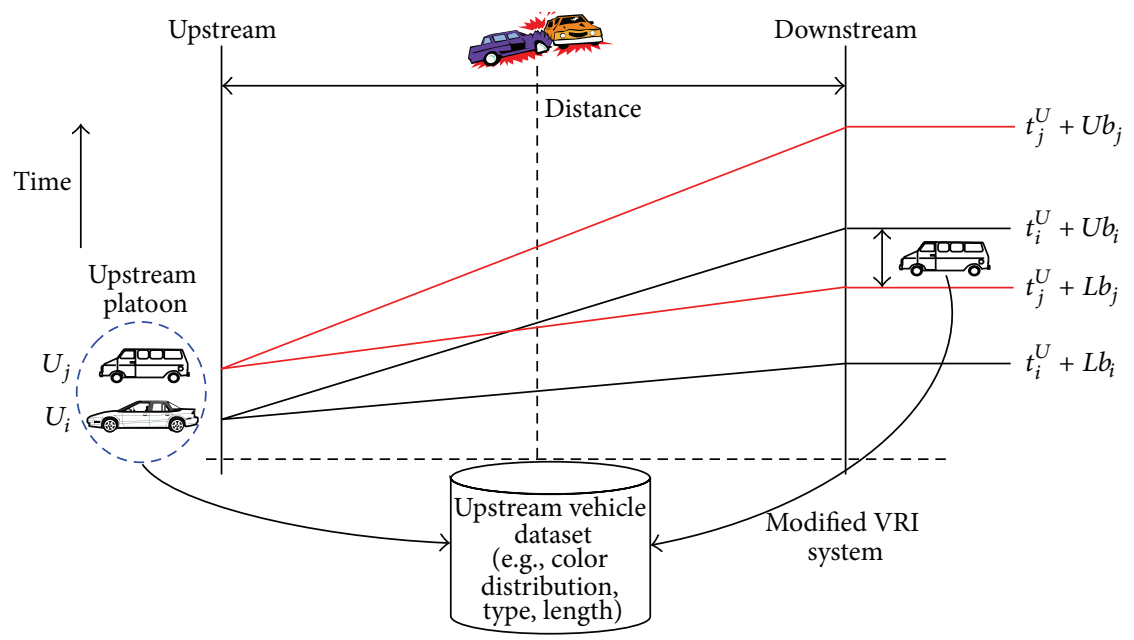

FIGURE 2: Illustrative example of vehicle count approach.

counts data, cannot promptly detect the incident (i.e., delay in incident detection). In general, the incident detection time would significantly increase with respect to the increase in size of vehicle platoon at the upstream detector, which increases the number of overlapping in arrival time intervals at the downstream detector.

To reduce the detection time, this research proposes a novel incident detection algorithm by incorporating the vision-based VRI system. As shown in Figure 2, vehicle $U_{i}$ and $U_{j}$ are detected and their detailed feature data (e.g., color, type and length) are also extracted. Once a vehicle is detected at downstream site, the proposed VRI system is performed to find a matched upstream vehicle based on the vehicle feature data. In the case that vehicle $U_{i}$ is missing, if the downstream vehicle could be matched to the vehicle $U_{j}$ based on the vehicle feature, an incident alarm would be triggered at time $t_{i}^{U}+U b_{i}$, as vehicle $U_{i}$ is not reidentified during time window $\left[t_{i}^{U}+L b_{i}, t_{i}^{U}+U b_{i}\right]$. As shown by this "toy" example, the additional VRI component could potentially reduce the incident detection time to some extent. However, it is also observed that the concept of VRI is not readily transferable to the field of incident detection (mismatches of VRI may trigger false alarms) and several modifications should be made regarding the vehicle matching process.

(i) First, instead of finding the matching result for upstream vehicle, the incident-detection-oriented VRI attempts to match the vehicles at downstream site such that the proposed AID algorithm can be implemented in real-time.

(ii) Second, once a vehicle passes the downstream station, the incident-detection-oriented VRI should be capable of making matching decision immediately such that the missing vehicle (i.e., the vehicle that does not appear at downstream) could be promptly identified. Therefore, this study calculates the matching probability for each pair of vehicles on which the following screening method could be imposed to further reduce the false alarm rate.
The overall framework of the proposed algorithm is presented in the following subsection.

3.2. AID Algorithm Based on VRI System. The detailed implementation of the VRI-based incident detection system is summarized in the following flowchart (Figure 3). First, the system will initialize the timestamp, $t$, and check whether a vehicle is detected at upstream and/or downstream station. If a vehicle is detected at upstream detector, the expected arrival time window of this vehicle at downstream station will be estimated based on the historical data. The record of the detected vehicle at upstream will be stored in the database as unmatched upstream vehicle. On the other hand, if a vehicle is captured at the downstream station, the system will perform incident-detection-oriented VRI subsystem to check whether this detected vehicle match with any of the unmatched upstream vehicle. The time window constraint is utilized to identify the potential matches for this vehicle detected at downstream station. Once the match is found, the matched vehicle data will be removed from the list of the unmatched upstream vehicles.

After the previous two steps for handling the detected vehicles at upstream and downstream stations, the system will proceed to determine whether there is an incident occurs on the monitored segment. For incident detection, the system will screen through the list of unmatched vehicles. If the current time $(t)$ is out of the expected arrival time window (i.e., greater than the upper bound of the arrival time interval) of the unmatched vehicle, an incident alarm will be issued. If not, $t$ will be set to $t+1$ and the system will move forward to the next time step. It could be easily observed that the performance of the incident detection system is heavily dependent on two critical components, that is, flexible time window constraint and incident-detection-oriented VRI system.

For the aforementioned framework, the following three comments should be taken into account.

(i) First, the detection error is not considered in this study. In other words, it is assumed that all the 


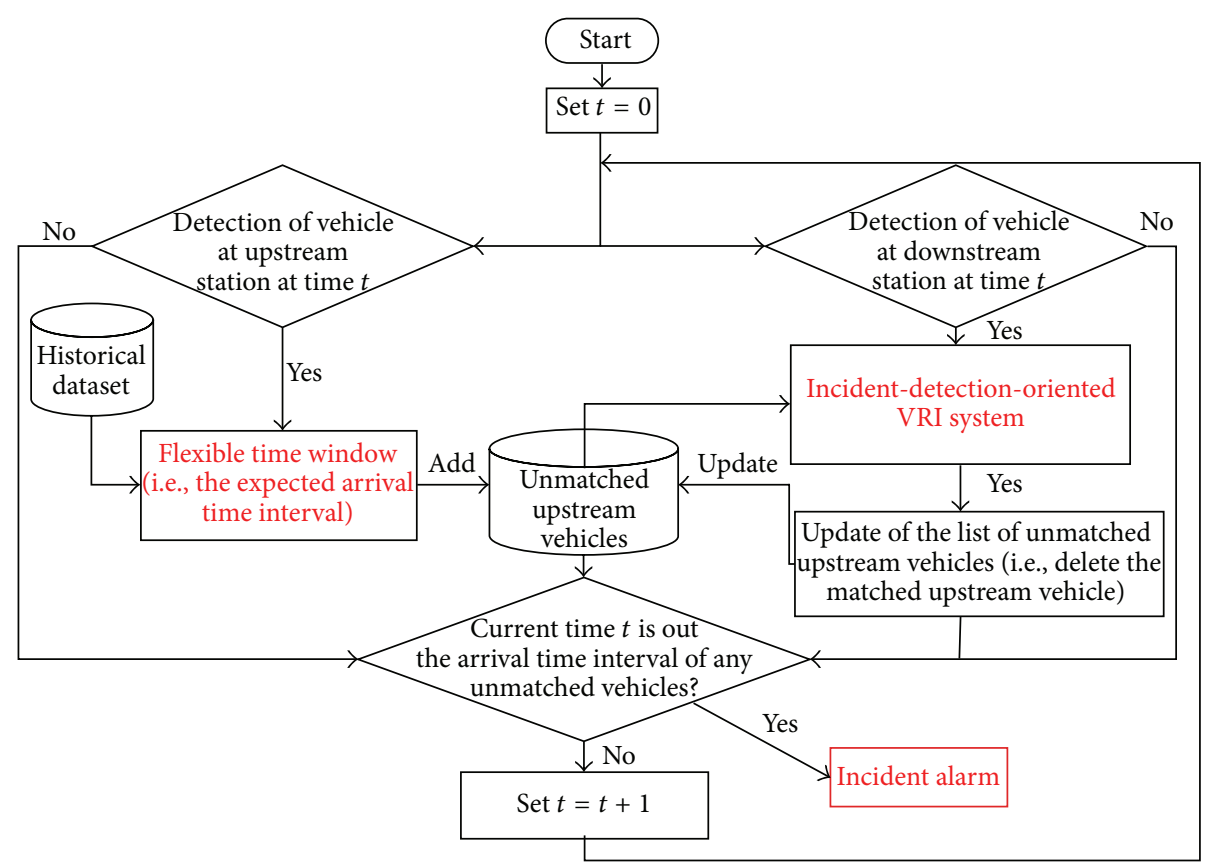

FIgURE 3: Overall framework of AID system.

vehicles cross the video cameras will be detected. This is achievable under free flow condition, as there is no occlusion between the vehicles and, consequently, VIPs perform generally well and are able to detect most of the individual vehicles.

(ii) Second, under free flow condition, the traveling behavior of the individual vehicle is more predictable. This phenomenon enables the estimation of the flexible arrival time window for each individual vehicle based on the current spot speed and the historical data. It is expected that the accurate estimation of the arrival time window could potentially lead to an improved matching accuracy of the VRI method, and hence reduce the incident detection time.

(iii) Third, it should be noted that the proposed VRI cannot guarantee an accurate matching because of the nonuniqueness of the vehicle signatures. Instead, the proposed VRI scheme in this paper can only provide the matching probability between the downstream and upstream vehicles. Therefore, some of the mismatches resulted from the matching probability could potentially lead to false alarms. To handle this, a ratio method is introduced to screen out those mismatches for reducing the false alarms.

\section{Flexible Time Window Estimation}

Under free flow condition, each individual vehicle would maintain in a relatively stable speed (i.e., low variance in travel time). In this case, the arrival time of the vehicle at downstream station could be estimated based on the spot speed and historical data. Let $U_{i}$ represent an upstream vehicle detected at time $t_{i}^{U}$, and the associated upstream spot speed is denoted as $v_{i}^{U}$. The expected arrival time Arr of vehicle $U_{i}$ is given by

$$
\operatorname{Arr}=t_{i}^{U}+\frac{l}{0.5\left(v_{i}^{U}+v_{i}^{D}\right)},
$$

where $l$ is the distance between the upstream and downstream detectors; $v_{i}^{D}$ is the estimated vehicle speed at downstream detector based on the historical speed database. To account for the error in estimating the downstream spot speed, the upper and lower bounds of $v_{i}^{D}$ are provided by the following equations:

$$
\begin{gathered}
v_{\mathrm{ub}}^{D}=\sigma_{\mathrm{ub}} V_{\text {hist }}^{D}\left(t^{\prime}\right) \frac{v_{i}^{U}}{V^{U}}, \\
v_{\mathrm{lb}}^{D}=\sigma_{\mathrm{lb}} V_{\text {hist }}^{D}\left(t^{\prime}\right) \frac{v_{i}^{U}}{V^{U}},
\end{gathered}
$$

where $v_{\mathrm{ub}}^{D}$ and $v_{\mathrm{lb}}^{D}$ are, respectively, the upper and lower bounds of the vehicle at downstream detector; $V^{U}$ is the current average speed of the upstream detector; $\sigma_{\mathrm{ub}} \geq 1$ and $\sigma_{\mathrm{lb}} \leq 1$ are respectively the associated upper and lower bound factors; $V_{\text {hist }}^{D}\left(t^{\prime}\right)$ is the historical average speed of the downstream detector at time $t^{\prime}$. The time $t^{\prime}$ is chosen such that it is matched with the arrival time, which is estimated by a linear speed profile of the modeled section, at the downstream detector. The estimation of downstream spot speeds can be viewed as a prediction-correction process. First, the historical average speed $V_{\text {hist }}^{D}\left(t^{\prime}\right)$ is adopted to predict the speed of this vehicle at downstream site. Then, this prediction is corrected by the factor $v_{i}^{U} / V^{U}$ for the better representation of the current traffic condition. Finally, the upper and lower bound factors $\left(\sigma_{\mathrm{ub}}\right.$ and $\left.\sigma_{\mathrm{lb}}\right)$ are applied for 
determining the upper of lower bounds of the downstream spot speed. With the estimated downstream speeds, the corresponding upper and lower bounds of the travel time of vehicle $U_{i}$ can be calculated as follows:

$$
\begin{aligned}
U b_{i} & =\frac{l}{0.5\left(v_{i}^{U}+v_{\mathrm{lb}}^{D}\right)}, \\
L b_{i} & =\frac{l}{0.5\left(v_{i}^{U}+v_{\mathrm{ub}}^{D}\right)} .
\end{aligned}
$$

However, it should be noted that the proposed incident detection system is not confined to the above method for estimating the time window. Any other estimation methods are equally applicable to the proposed AID algorithm. With the estimated time windows, vehicles on the monitored freeway section could be "partially" tracked and reidentified in a timely and accurate manner.

\section{Incident-Detection-Oriented VRI}

As explained previously, the proposed VRI system is devised based on the video image data provided by VIPs technology. By applying myriad image processing techniques, the detailed vehicle feature data (e.g., color, type, and length) could be obtained. The vehicle matching process is then performed by comparing these vehicle feature data. In this section, the methodologies involved in the incident-detection-oriented VRI system are presented.

5.1. Vehicle Matching Problem. For a vehicle $D_{k}$ arrives at downstream station at time $t_{k}^{D}$, the vehicle signature, denoted as $X_{k}^{D}=\left\{C_{k}^{D}, S_{k}^{D}, L_{k}^{D}\right\}$, is then obtained from the VIPs. A search space, $\delta(k)$, which represents the potential matches at upstream station for vehicle $D_{k}$, is determined based on the calculated arrival time window. Specifically, $\delta(k)$ is given by

$$
\mathcal{S}(k)=\left\{U_{i} \in U \mid t_{i}^{U}+L b_{i} \leq t_{k}^{D} \leq t_{i}^{U}+U b_{i}\right\},
$$

where $U_{i}$ represents the vehicle detected at upstream station; $\left[L b_{i}, U b_{i}\right]$ is the associated travel time window. The vehicle reidentification problem is to find the corresponding upstream vehicle for $D_{k}$ through the search space $\delta(k)$. Herein, we introduce the assignment function $\psi$ to represent the matching result; that is,

$$
\begin{aligned}
& \psi(k): \\
& \left\{\begin{array}{l}
D_{k} \longrightarrow\left\{U_{i} \in \mathcal{S}(k) \mid i=1,2, \ldots, N\right\} \\
k \longmapsto i,
\end{array} \quad i=1,2, \ldots, N,\right.
\end{aligned}
$$

where $\psi(k)=i$ indicates that vehicle $D_{k}$ is the same as $U_{i}$. Recall that for each vehicle $U_{i} \in S(k)$, one may assign to the pair of signatures $\left(X_{i}^{U}, X_{k}^{D}\right)$ the distances $d_{\text {color }}(i, k)$, $d_{\text {type }}(i, k)$ and $d_{\text {length }}(i, k)$ based on (3), (4), and (5). In this case, one simple method (i.e., distance-based method) is to find the matched upstream vehicle with the minimum feature distance. However, it should be noted that the vehicle signatures derived from VIPs contain noise and are not unique. Therefore, the distance measure cannot really reflect the similarities between the vehicles. Instead of directly comparing the feature distances, this study utilizes the statistical matching method. Based on the calculated feature distances, that is, $d_{\text {color }}(i, k), d_{\text {type }}(i, k)$, and $d_{\text {length }}(i, k)$, a matching probability $P\left(\psi(k)=i \mid d_{\text {color }}, d_{\text {type }}, d_{\text {length }}\right)$ between vehicles $U_{i}$ and $D_{k}$ is provided for the matching decision making.

5.2. Calculation of Matching Probability. The matching probability, also referred to as the posterior probability, plays a fundamental role in the proposed VRI system. By applying the Bayesian rule, we have

$$
\begin{aligned}
& P\left(\psi(k)=i \mid d_{\text {color }}, d_{\text {type }}, d_{\text {length }}\right) \\
& =\frac{p\left(d_{\text {color }}, d_{\text {type }}, d_{\text {length }} \mid \psi(k)=i\right) P(\psi(k)=i)}{p\left(d_{\text {color }}, d_{\text {type }}, d_{\text {length }}\right)},
\end{aligned}
$$

where $p\left(d_{\text {color }}, d_{\text {type }}, d_{\text {length }} \mid \psi(k)=i\right)$ is the likelihood function; $P(\psi(k)=i)$ is the prior knowledge of the assignment function. To obtain the explicit matching probability, the denominator in (11) can further be expressed as

$$
\begin{aligned}
& p\left(d_{\text {color }}, d_{\text {type }}, d_{\text {length }}\right) \\
& =p\left(d_{\text {color }}, d_{\text {type }}, d_{\text {length }} \mid \psi(k)=i\right) P\left(\psi_{k}=i\right) \\
& \quad+p\left(d_{\text {color }}, d_{\text {type }}, d_{\text {length }} \mid \psi(k) \neq i\right) P\left(\psi_{k} \neq i\right) .
\end{aligned}
$$

On the basis of (11) and (12), it is easily observed that the calculation of the matching probability is dependent on the deduction of the likelihood function and the prior probability. In this particular case, the prior probability is defined as $P(\psi(k)=i)=0.5$, which suggests that the matching is solely based on the comparison between the vehicle feature data. The calculation of the likelihood function is completed in two steps.

(i) First, individual statistical models for the three feature distances are constructed and the corresponding likelihood functions are also obtained (i.e., $p\left(d_{\text {color }} \mid\right.$ $\psi(k)), p\left(d_{\text {type }} \mid \psi(k)\right)$, and $\left.p\left(d_{\text {length }} \mid \psi(k)\right)\right)$.

(ii) Second, a data fusion rule is employed to provide the overall likelihood functions, that is, the term $p\left(d_{\text {color }}, d_{\text {type }}, d_{\text {length }} \mid \psi(k)\right)$ in (11) and (12).

5.2.1. Statistical Modeling of Feature Distance. Without loss of generality, only the probabilistic modeling of color feature distance is described. In the framework of statistical modeling, the distance measure is assumed to be a random variable. Thus, for a pair of color feature vectors $\left(C_{i}^{U}, C_{k}^{D}\right)$, the distance $d_{\text {color }}(i, k)$ follows a certain statistical distribution. The conditional probability (i.e., likelihood function) of $d_{\text {color }}(i, k)$ is then given by

$$
\begin{aligned}
p\left(d_{\text {color }}(i, k) \mid \psi(k)\right) & \begin{cases}p_{1}\left(d_{\text {color }}(i, k)\right), & \text { if } \psi(k)=i \\
p_{2}\left(d_{\text {color }}(i, k)\right), & \text { if } \psi(k) \neq i,\end{cases}
\end{aligned}
$$




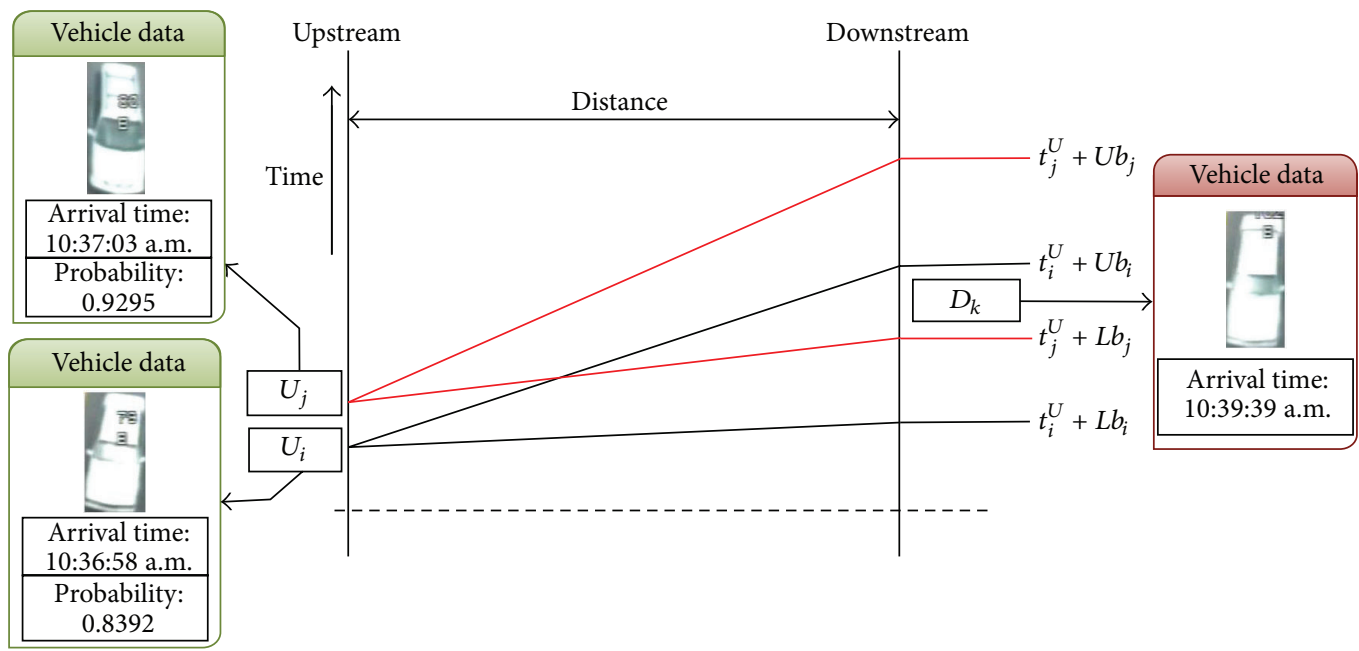

Figure 4: Illustrative example of a false alarm.

where $p_{1}$ denotes the probability density function (pdf) of distance $d_{\text {color }}(i, k)$ when color feature vectors $C_{i}^{U}$ and $C_{k}^{D}$ belong to the same vehicle, while $p_{2}$ is the pdf of the distance $d_{\text {color }}(i, k)$ between different vehicles. A historical training dataset that contains a number of pairs of correctly matched vehicles is built up for estimating the pdfs $p_{1}$ and $p_{2}$. Finite Gaussian mixture model is used to approximate the pdfs and the well-known Expectation Maximization (EM) algorithm is applied to solve the associated parameter estimation problem. Likewise, the likelihood functions for the type and length distances can also be obtained in a similar manner.

5.2.2. Data Fusion Rule. In this study, the logarithmic opinion pool (LOP) approach is employed to fuse the individual likelihood functions. The LOP is evaluated as a weighted product of the probabilities and the equation is given by

$$
\begin{gathered}
p\left(d_{\text {color }}, d_{\text {type }}, d_{\text {length }} \mid \psi(k)\right)=\frac{1}{Z_{\mathrm{LOP}}} \\
\cdot p\left(d_{\text {color }} \mid \psi(k)\right)^{\alpha} p\left(d_{\text {type }} \mid \psi(k)\right)^{\beta} \\
\cdot p\left(d_{\text {length }} \mid \psi(k)\right)^{\gamma}, \quad \alpha+\beta+\gamma=1,
\end{gathered}
$$

where the fusion weights, $\alpha, \beta$, and $\gamma$ are used to indicate the degree of contribution of each likelihood function. The weights can also be calibrated from the training dataset. By substituting (12), (13), and (14) into (11), the desired matching probability for each pair of vehicles $\left(U_{i}, D_{k}\right)$ could be obtained. For the sake of simplicity, let $P_{i k}$ denote the matching probability between the vehicle $U_{i}$ and $D_{k}$. In this case, we may obtain a set of probabilistic measures $\left\{P_{i k} \mid\right.$ $i=1,2, \ldots, N\}$ to represent the likelihood of a correct match between $D_{k}$ and the vehicles in the search space $\delta(k)$. The final matching decision-making based on these matching probabilities, becomes the major concern in the following subsection.
5.3. Ratio Method for Final Matching Decision. An intuitive decision-making process (i.e., the greedy method) is to sort the matches via the matching probability $\left\{P_{i k} \mid i=\right.$ $1,2, \ldots, N\}$ and choose the vehicle $U_{i}$ with the maximum matching likelihood; that is,

$$
\psi(k)=i, \quad \text { if } P_{j k} \leq P_{i k} \forall j \in\{1,2, \ldots, N\} .
$$

However, it is noteworthy that the proposed VRI system is utilized for incident detection purpose, the final matching decision would produce significant impacts on the performance of the AID system. Based on the greedy method (15), the potential false alarms would be triggered. As shown in Figure 4, the downstream vehicle $D_{k}$ arrives at time 10:39:39 a.m. $U_{j}$ and $U_{i}$ are, respectively, the two candidate vehicles with the largest and second largest matching probabilities with the downstream vehicle $D_{k}$ (i.e., $P_{j k}=0.9295$ and $P_{i k}=$ $0.8392)$. Although vehicle $D_{k}$ actually matches with vehicle $U_{i}$ (based on the manual matching), the greedy method yields the matching result $\psi(k)=j$, which could lead to a false alarm at time $t_{i}^{U}+U b_{i}$.

To reduce the false alarms mentioned above, a ratio method is then introduced for the final matching decisionmaking. Let $\left\{P_{i} \mid i=1,2, \ldots, N\right\}$ denote the set of matching probabilities in descending order. The ratio method proposed in this study involves two major steps. First, by imposing a threshold $\tau$ on the value of the ratio between the neighboring probabilities in the ordered set $\left\{P_{i} \mid i=1,2, \ldots, N\right\}$, one may be able to screen through the search space and rule out those unlikely matches. The screening process is described in Procedure 1.

The underlying implication of Procedure 1 is that if the ratio (i.e., $P_{i} / P_{i+1}$ ) is sufficiently large, then it could come to a conclusion that vehicles $\{i+1, i+2, \ldots, N\}$ are the unlikely matches due to their relatively smaller matching probabilities. Otherwise, if the ratio $P_{i} / P_{i+1} \leq \tau$, then we may declare that vehicle $i$ and $i+1$ are not distinctive from each other and a matching decision cannot be made at current stage. 
Input: A finite set $\left\{P_{i} \mid i=1,2, \ldots, N\right\}$ of matching probabilities in descending order Output: The set of unlikely matches for downstream vehicle $D_{k}$

(1) $i \leftarrow 1$;

(2) while $i \leq N-1 \wedge P_{i} / P_{i+1} \leq \tau$ do

(3) $\quad i \leftarrow i+1$;

(4) return $\{i+1, i+2, \ldots, N\}$;

Procedure 1: Algorithmic framework for screening process.

Input: A set $\left\{P_{i} \mid i=1,2, \ldots, N\right\}$ of matching probabilities and the set $\left\{t_{i}^{U}+U b_{i} \mid i=1,2, \ldots, N\right\}$ of upper bounds in arrival time interval

Output: The final matching decision for vehicle $D_{k}$

Screening Method:

(1) $i \leftarrow 1$;

(2) while $i \leq N-1 \wedge P_{i} / P_{i+1} \leq \tau$ do

(3) $\quad i \leftarrow i+1$;

(4) $\mathcal{S}_{R}(k) \leftarrow\{1,2, \ldots, i\}$;

Vehicle Count Approach:

(5) $m^{*} \leftarrow \arg \min _{l}\left\{t_{l}^{U}+U b_{l} \mid l \in \mathcal{S}_{R}(k)\right\}$;

(6) return $\psi(k)=m^{*}$;

Procedure 2: Algorithmic framework for final matching decision-making.

Upon the completion of the above screening process, unlikely matches could be ruled out and the search space is further reduced. The second step is then to make a matching decision based on the remaining search space $\mathcal{S}_{R}(k)$. Let $\mathcal{S}_{R}(k)=\left\{U_{m} \mid m=1,2, \ldots, i\right\}$ (clearly $\left.i \leq N\right)$, the matching result is then given by

$$
\begin{aligned}
& \psi(k)=m^{*}, \\
& \quad \text { if } t_{l}^{U}+U b_{l} \geq t_{m^{*}}^{U}+U b_{m^{*}}, \forall l \in\{1,2, \ldots, i\} .
\end{aligned}
$$

It is obvious that vehicle $D_{k}$ is matched to the vehicle in $\mathcal{S}_{R}(k)$ with the smallest upper bound in the predicted arrival time window. The rationale behind this approach is that a matching decision could not be made based on the matching probabilities (as the matching probabilities for vehicles in $\delta_{R}(k)$ are not significantly different from each other). In this case, the vehicle $D_{k}$ is matched to upstream vehicle with smallest upper bound in the predicted arrival time window to avoid the potential false alarms. As a matter of fact, the second step could be viewed as a standard vehicle count approach in which only the counts data is utilized.

To sum up, the matching decision-making process of the incident-detection-oriented VRI is a hybrid of the vehicle feature matching and the classic vehicle count approach. The overall procedure for the matching decision-making is given by Procedure 2 .

\section{Test Results}

In this section, the performance of the proposed AID algorithm is evaluated against the classical vehicle count approach in terms of mean time-to-detect and false alarm rate (i.e., false alarms per hour). As the performance of the proposed AID system relies on its two critical components (i.e., flexible time window estimation and incident-detectionoriented VRI), different sizes of time window and thresholds for final matching are tested in this section. The dataset described in Section 2 are used to perform the simulated tests for the algorithm evaluation. Also, the real-world case studies are carried out in this section.

6.1. Simulated Tests. For calibrating and testing the proposed AID system, the 3,682 pairs of vehicle matching results from the collected dataset are divided into two parts. First, a dataset of 800 pairs of correctly matched vehicles are used for model calibration and training. The upper and lower bound factors for time window estimation (i.e., $\sigma_{\mathrm{ub}}$ and $\sigma_{\mathrm{lb}}$ ) are calibrated by using the travel time data of the 800 vehicles and the historical averaging speed on Thursday, which is the same as the test day (i.e., 16/2/2012, 23/2/2012, 1/3/2012, and $8 / 3 / 2012$ ). In addition, the parameters of the statistical model (i.e., $p_{1}$ and $p_{2}$ ) are estimated by utilizing the feature data extracted from the captured images of these 800 pairs of vehicles. Second, the remaining 2,828 pairs of vehicles detected at both upstream and downstream detectors are fed into the calibrated AID system for model evaluation. In order to mimic an incident between the upstream and downstream detectors, the record of vehicle at downstream site is intentionally removed to simulate the situation that the vehicle has passed the upstream detector but not the downstream one. In the testing of the proposed AID system, the AID algorithm is run for 2,828 times, which for each run the record of one of the 2,828 vehicles at downstream 


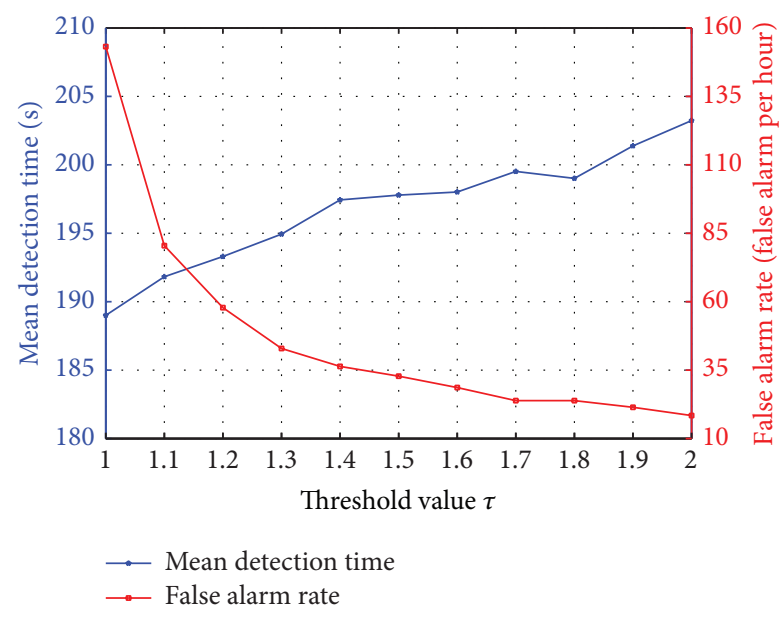

Figure 5: Mean detection time and false alarm rate.

detector is removed, for determining the mean detection time. Specifically, the incident detection time is defined as

$$
T_{D}=t_{\text {incident }}-t^{U} .
$$

Since we do not know the exact time when the incident happened, the incident detection time is then defined as the difference between the time when an alarm is issued (i.e., $\left.t_{\text {incident }}\right)$ and the arrival time of incident vehicle at upstream station (i.e., $t^{U}$ ).

By setting the threshold value equals to 2 (i.e., $\tau=2$ ), the mean detection time of the proposed AID algorithm is 203.2 seconds, whereas the mean detection time of the classical vehicle count approach is 644.1 seconds. As it is expected, the mean detection time is reduced substantially by incorporating the modified VRI system. Figure 5 shows the performance of the VRI-based incident detection algorithm for different threshold values adopted in final matching decision. It could be observed that the false alarm rate reduces as the threshold value increases. When the threshold value equals one, the VRI system will always match the downstream vehicle to the upstream one with the largest matching probability. Therefore, it would lead to a large number of false alarms (see Section 5.3). With the increase in threshold value, the modified VRI system relies more on the traditional vehicle count approach and results in a decrease in the false alarm rate. On the other hand, as the proposed VRI system is more relied on the traditional vehicle count approach (e.g., $\tau \rightarrow \infty$ ), the mean detection time also increases (see Section 3.1). To sum up, for the proposed VRI system, the lowering of the false alarm rates is at the expense of incident detection time. Thus, a balance should be struck between the rapid incident detection and low false alarm rate.

The estimation of arrival time window also has a significant impact on the performance of the proposed AID algorithm. It is not difficult to understand that a small time window size would result in faster incident detection. To test the performance of the proposed AID algorithm under different time window sizes, a time window with fixed size is assigned for each individual vehicle. Figure 6 shows the

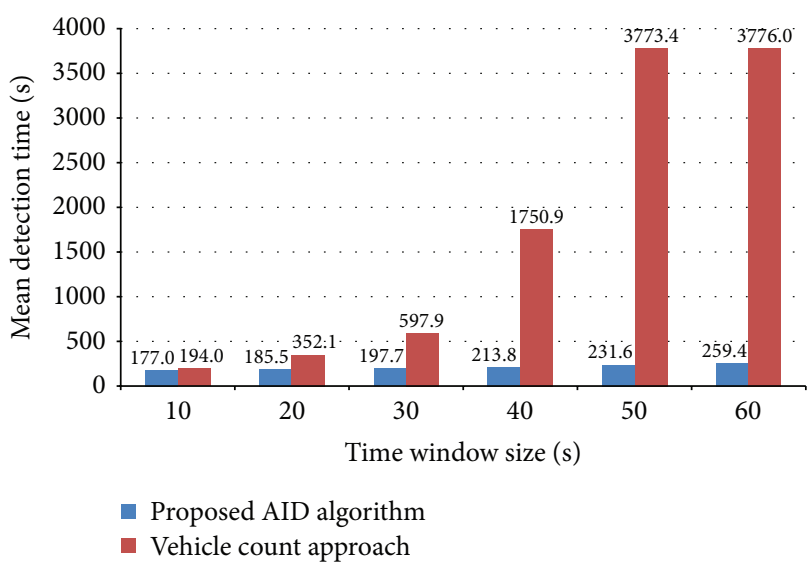

FIgURE 6: Comparison between the proposed AID algorithm and vehicle count approach.

mean detection of the algorithm for different time window sizes. The mean detection time of the vehicle count approach increases dramatically as the size of the time window grows. It is also observed that the vehicle count approach is not capable of detecting the missing vehicle as the size of time window is larger than 50 seconds. To sum up, for the simulation that a large arrival time window is applied, the proposed AID algorithm clearly outperforms the vehicle count approach.

6.2. Real-World Case Studies. Apart from the abovementioned simulation tests, two real-world case studies are also carried out. Based on the record from the freeway authority, the first incident is reported on June 13, 2012, at 16:03. The reported incident location is at $20+600$ westbound, which is in the section between camera $7 \mathrm{~A} / 8 \mathrm{~A}$ and $9 \mathrm{~A} / 10 \mathrm{~A}$ (see Figure 1). Based on this information, the research team has screened through the captured videos for identifying the incident vehicle. It is found out that on June 13, 2012, the incident vehicle has passed the upstream detector (7A/8A) at 15:55 (Figure 7(a)) and has an incident before it reaches the downstream detector $(9 \mathrm{~A} / 10 \mathrm{~A})$. Four minutes later, a tow truck, which is probably called by the driver of the incident vehicle, has passed the upstream detector (Figure 7(b)) and towed the incident vehicle to pass the downstream detector at 16:09 Figure 7(c)).

According to the above information of the incident vehicle, a 35-minute video record data (from 15:33 to 16:08 on June 13,2012 ) of locations $8 \mathrm{~A}$ and $10 \mathrm{~A}$ are extracted and input into the proposed AID system for free flow condition. In this case, apart from the incident vehicle, 739 vehicles are detected at both stations during the 35 -minute video record. By setting the threshold value of the ratio of matching probabilities equals to 8.5 , the time of incident detection and the false alarm rate for this case study are found to be 15:58:22, and 3.42 false alarms per hour, respectively. Compared with the classic vehicle count approach, which would trigger an incident alarm at 16:01:28, the proposed AID system performs better in terms of the incident detection time.

The incident vehicle of the second real-world case study is shown in Figure 8. This incident is reported on June 17, 


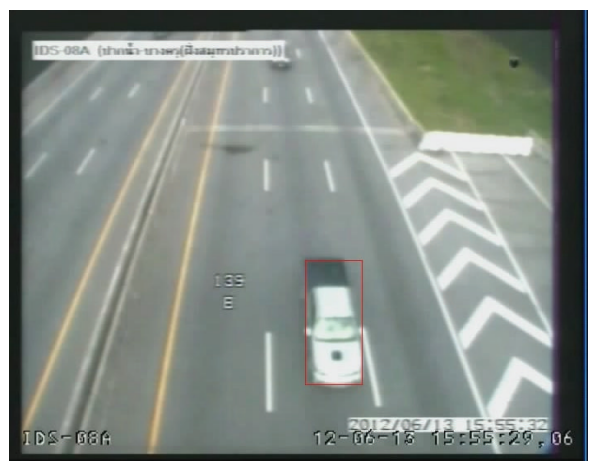

(a)

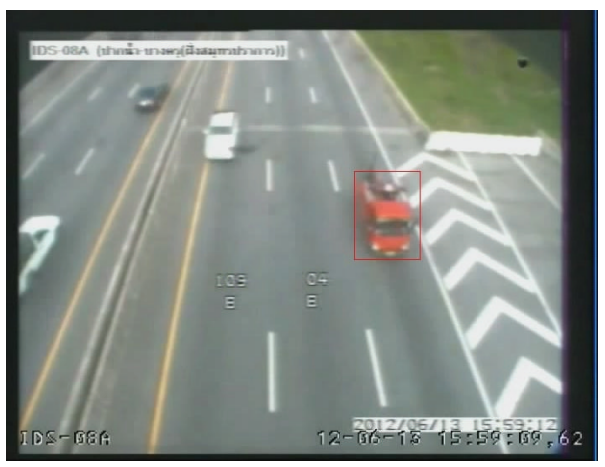

(b)

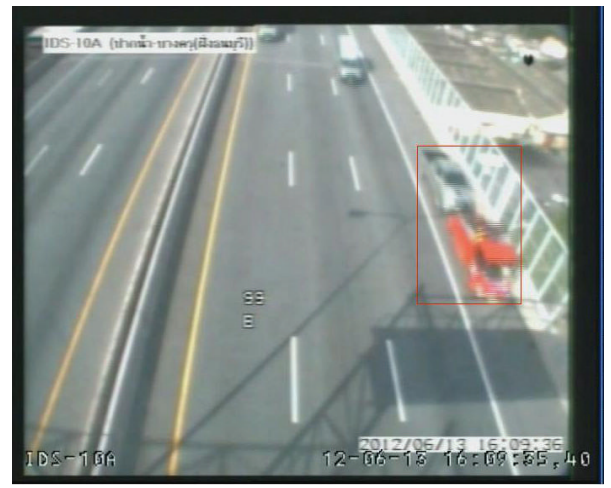

(c)

FIGURE 7: Real-world case study \#1: (a) incident vehicle passes the upstream detector; (b) tow truck passes the upstream detector; (c) incident vehicle and truck passes through the downstream detector.

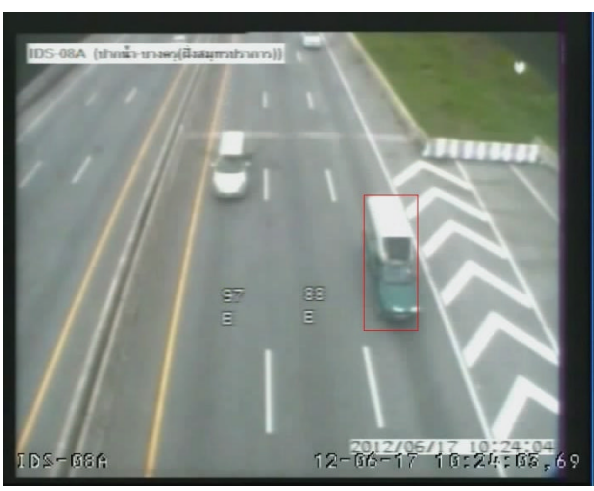

(a)

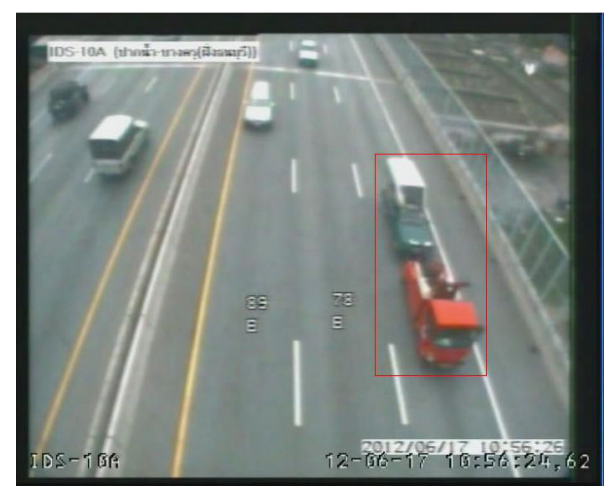

(b)

Figure 8: Real-world case study \#2: (a) incident vehicle passes the upstream detector; (b) incident vehicle and truck passes through the downstream detector.

2012 at 10:31 a.m. and its detailed location is at $19+300 \mathrm{~A}$ westbound (between $7 \mathrm{~A} / 8 \mathrm{~A}$ and $9 \mathrm{~A} / 10 \mathrm{~A}$ ). By setting the threshold value of the ratio of matching probabilities equal to 8.5 , the time of incident detection and the false alarm rate for this case study are found to be 10:28:22 and 2 false alarms per hour, respectively. Compared with the classic vehicle count approach, which would trigger an incident alarm at 10:33:50, the proposed AID system still performs better in terms of the incident detection time.
On the basis of these two real-world case studies, we may observe that the time of incident detection of the proposed AID algorithm is largely dependent on the actual information associated with the incident vehicle (e.g., distinctiveness of the incident vehicle feature and the size of the vehicle platoon). In real-world case study \#2, the incident vehicle has "distinctive" vehicle color distribution (see Figure 8(a)) and, consequently, the disappearance of this very vehicle can be identified earlier than the vehicle count approach. The size 
of vehicle platoon may also have significant impact on the performance of the AID algorithm. The larger the platoon size is, the more likely it is that the arrival time windows of these vehicles may overlap each other at the downstream site and, hence, leads to the significant increase in incident detection time.

\section{Conclusion and Future Works}

This paper investigates the feasibility of utilizing the vehicle reidentification system for incident detection on a freeway section under the free flow condition. A modified vision-based VRI system is proposed to partially track the individual vehicle for identifying the "missing" vehicle due to an incident. A flexible arrival time window is estimated for each of the individual vehicle at upstream station to improve the matching accuracy. To reduce the potential false alarms, a screening method, which is based on the ratios of the matching probabilities and arrival time windows, is introduced to rule out the potential mismatches.

The proposed AID algorithm is tested on a $3.6 \mathrm{~km}$ segment of a closed freeway in Bangkok, Thailand. Based on the test results, it is found out that the detection time of the proposed AID algorithm is substantially shorter than the traditional vehicle count approach. Also, there is a trade-off between the false alarms rate and detection time for the proposed AID algorithm. Therefore, a balance should be struck between the rapid incident detection and low false alarm rate by adjusting the thresholding value $\tau$. As demonstrated in Procedure 2, the proposed AID algorithm is a hybrid of the vehicle feature comparison method and the classical vehicle count approach, and the threshold value $\tau$ can be viewed as a switch between these two methods. Therefore, the selection of $\tau$ may be of great importance to the proposed AID algorithm. In this study, we adjust the threshold value manually based on the reliability of VRI system (the performance of the VRI system in different time period may be slightly different due to the changes in outdoor environment, and the threshold value $\tau$ should be adjusted accordingly). Some other automatic thresholding processes [29] will be investigated in our future works.

Note that the proposed AID algorithm is specifically devised to detection incident on freeway system under freeflow conditions. As a natural and necessary extension, the ability of detecting incidents under dynamic traffic condition is required for the further development of incident detection system. The key component would be to devise an additional VRI based detection algorithm for congested situation. Theoretically, this purpose can be achieved by analyzing the temporal changes in travel time information obtained from VRI system under dynamic traffic conditions [25].

\section{Conflict of Interests}

The authors declare that there is no conflict of interests regarding the publication of this paper.

\section{Acknowledgment}

This work was supported by the Hong Kong Research Grants Council under General Research Fund PolyU 5242/12E.

\section{References}

[1] Y. B. Ji, R. Jiang, M. Qu, and E. Chung, "Traffic incident clearance time and arrival time prediction based on hazard models," Mathematical Problems in Engineering, vol. 2014, Article ID 508039, 11 pages, 2014.

[2] C.-S. Chou and E. Miller-Hooks, "Simulation-based secondary incident filtering method," Journal of Transportation Engineering, vol. 136, no. 8, pp. 746-754, 2010.

[3] E. I. Vlahogianni, M. G. Karlaftis, and F. P. Orfanou, "Modeling the effects of weather and traffic on the risk of secondary incidents," Journal of Intelligent Transportation Systems: Technology, Planning, and Operations, vol. 16, no. 3, pp. 109-117, 2012.

[4] H. J. Payne and S. C. Tignor, "Freeway incident detection algorithms based on decision tree with states," Transportation Research Record, vol. 682, pp. 30-37, 1978.

[5] F. L. Hall, Y. Shi, and G. Atala, "On-line testing of the mcmaster incident detection algorithm under recurrent congestion," Transportation Research Record, no. 1394, pp. 1-7, 1993.

[6] D. Srinivasan, X. Jin, and R. L. Cheu, "Evaluation of adaptive neural network models for freeway incident detection," IEEE Transactions on Intelligent Transportation Systems, vol. 5, no. 1, pp. 1-11, 2004.

[7] K. Zhang and M. A. P. Taylor, "Effective arterial road incident detection: a bayesian network based algorithm," Transportation Research Part C: Emerging Technologies, vol. 14, no. 6, pp. 403417, 2006.

[8] Q. Liu, J. Lu, S. Chen, and K. Zhao, "Multiple Naïve bayes classifiers ensemble for traffic incident detection," Mathematical Problems in Engineering, vol. 2014, Article ID 383671, 16 pages, 2014.

[9] C. Zheng, S. Chen, W. Wang, and J. Lu, "Using principal component analysis to solve a class imbalance problem in traffic incident detection," Mathematical Problems in Engineering, vol. 2013, Article ID 524861, 8 pages, 2013.

[10] A. Hofleitner, R. Herring, P. Abbeel, and A. Bayen, "Learning the dynamics of arterial traffic from probe data using a dynamic bayesian network," IEEE Transactions on Intelligent Transportation Systems, vol. 13, no. 4, pp. 1679-1693, 2012.

[11] G. Rose, "Mobile phones as traffic probes: practices, prospects and issues," Transport Reviews, vol. 26, no. 3, pp. 275-291, 2006.

[12] M. S. Shehata, J. Cai, W. M. Badawy et al., "Video-based automatic incident detection for smart roads: the outdoor environmental challenges regarding false alarms," IEEE Transactions on Intelligent Transportation Systems, vol. 9, pp. 349-360, 2008.

[13] H. Shao, W. H. K. Lam, A. Sumalee, and A. Chen, "Journey time estimator for assessment of road network performance under demand uncertainty," Transportation Research Part C: Emerging Technologies, vol. 35, pp. 244-262, 2013.

[14] D. B. Fambro and G. P. Ritch, "Evaluation of an algorithm for detecting urban freeway incidents during low-volume conditions," Transportation Research Record, vol. 773, pp. 31-39, 1980.

[15] S.-L. Chang, L.-S. Chen, Y.-C. Chung, and S.-W. Chen, "Automatic license plate recognition," IEEE Transactions on Intelligent Transportation Systems, vol. 5, no. 1, pp. 42-53, 2004. 
[16] S. M. Quayle, P. Koonce, D. Depencier, and D. M. Bullock, "Arterial performance measures with media access control readers: Portland, Oregon, pilot study," Transportation Research Record, no. 2192, pp. 185-193, 2010.

[17] C. Sun and S. G. Ritchie, "Individual vehicle speed estimation using single loop inductive waveforms," Journal of Transportation Engineering, vol. 125, no. 6, pp. 531-538, 1999.

[18] B. Coifman and S. Krishnamurthy, "Vehicle reidentification and travel time measurement across freeway junctions using the existing detector infrastructure," Transportation Research Part C: Emerging Technologies, vol. 15, no. 3, pp. 135-153, 2007.

[19] S. Kamijo, T. Kawahara, and M. Sakauchi, "Vehicle sequence image matching for travel time measurement between intersections," in Proceedings of the IEEE International Conference on Systems, Man and Cybernetics, vol. 2, pp. 1359-1364, IEEE, Waikoloa, Hawaii, USA, October 2005.

[20] C. C. Sun, G. S. Arr, R. P. Ramachandran, and S. G. Ritchie, "Vehicle reidentification using multidetector fusion," IEEE Transactions on Intelligent Transportation Systems, vol. 5, no. 3, pp. 155-164, 2004.

[21] M. Cetin, C. M. Monsere, and A. P. Nichols, "Bayesian models for reidentification of trucks over long distances on the basis of axle measurement data," Journal of Intelligent Transportation Systems: Technology, Planning, and Operations, vol. 15, no. 1, pp. $1-12,2011$.

[22] B. Coifman, "Vehicle re-identification and travel time measurement in real-time on freeways using existing loop detector infrastructure," Transportation Research Record, no. 1643, pp. 181-191, 1998.

[23] K. Kwong, R. Kavaler, R. Rajagopal, and P. Varaiya, "Arterial travel time estimation based on vehicle re-identification using wireless magnetic sensors," Transportation Research Part C: Emerging Technologies, vol. 17, no. 6, pp. 586-606, 2009.

[24] A. Sumalee, J. Wang, K. Jedwanna, and S. Suwansawat, "Probabilistic fusion of vehicle features for reidentification and travel time estimation using video image data," Transportation Research Record, vol. 2308, pp. 73-82, 2012.

[25] J. Wang, N. Indra-Payoong, A. Sumalee, and S. Panwai, "Vehicle reidentification with self-adaptive time windows for real-time travel time estimation," IEEE Transactions on Intelligent Transportation Systems, vol. 15, no. 2, pp. 540-552, 2014.

[26] C. Mallikarjuna, A. Phanindra, and K. R. Rao, "Traffic data collection under mixed traffic conditions using video image processing," Journal of Transportation Engineering, vol. 135, no. 4, pp. 174-182, 2009.

[27] A. T. G. Thiang and R. Lim, "Type of vehicle recognition using template matching method," in Proceedings of the International Conference on Electrical, Electronics, Communication and Information, pp. 7-8, Jakarta, Indonesia, 2001.

[28] A. Bhattacharyya, "On a measure of divergence between two statistical populations defined by their probability distributions," Bulletin of the Calcutta Mathematical Society, vol. 35, pp. 99-109, 1943.

[29] N. Otsu, "A threshold selection method from gray-level histograms," IEEE Trans Syst Man Cybern, vol. 9, no. 1, pp. 62-66, 1979. 


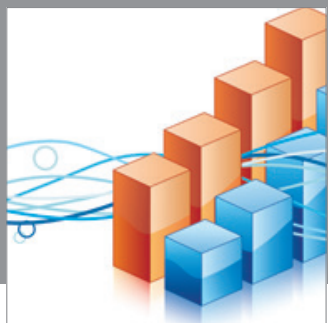

Advances in

Operations Research

mansans

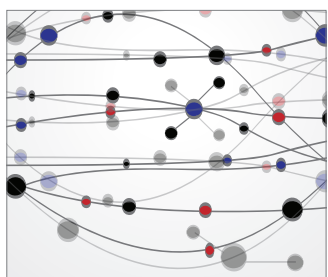

The Scientific World Journal
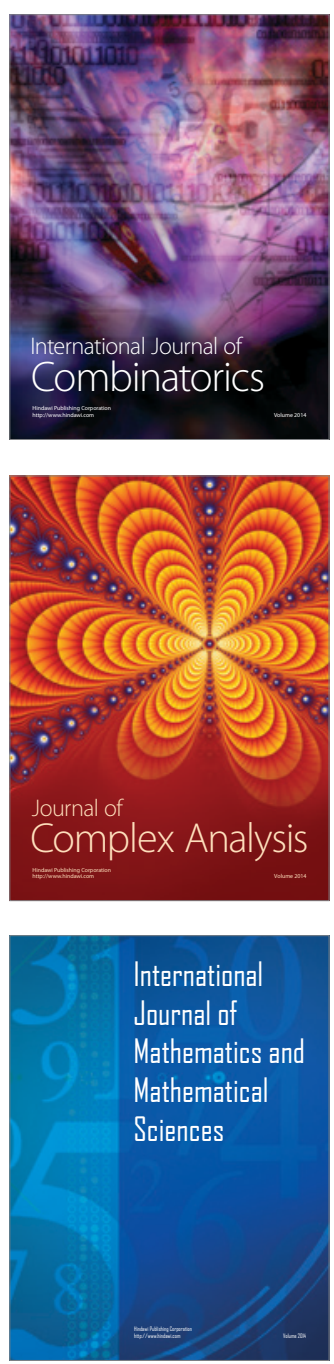
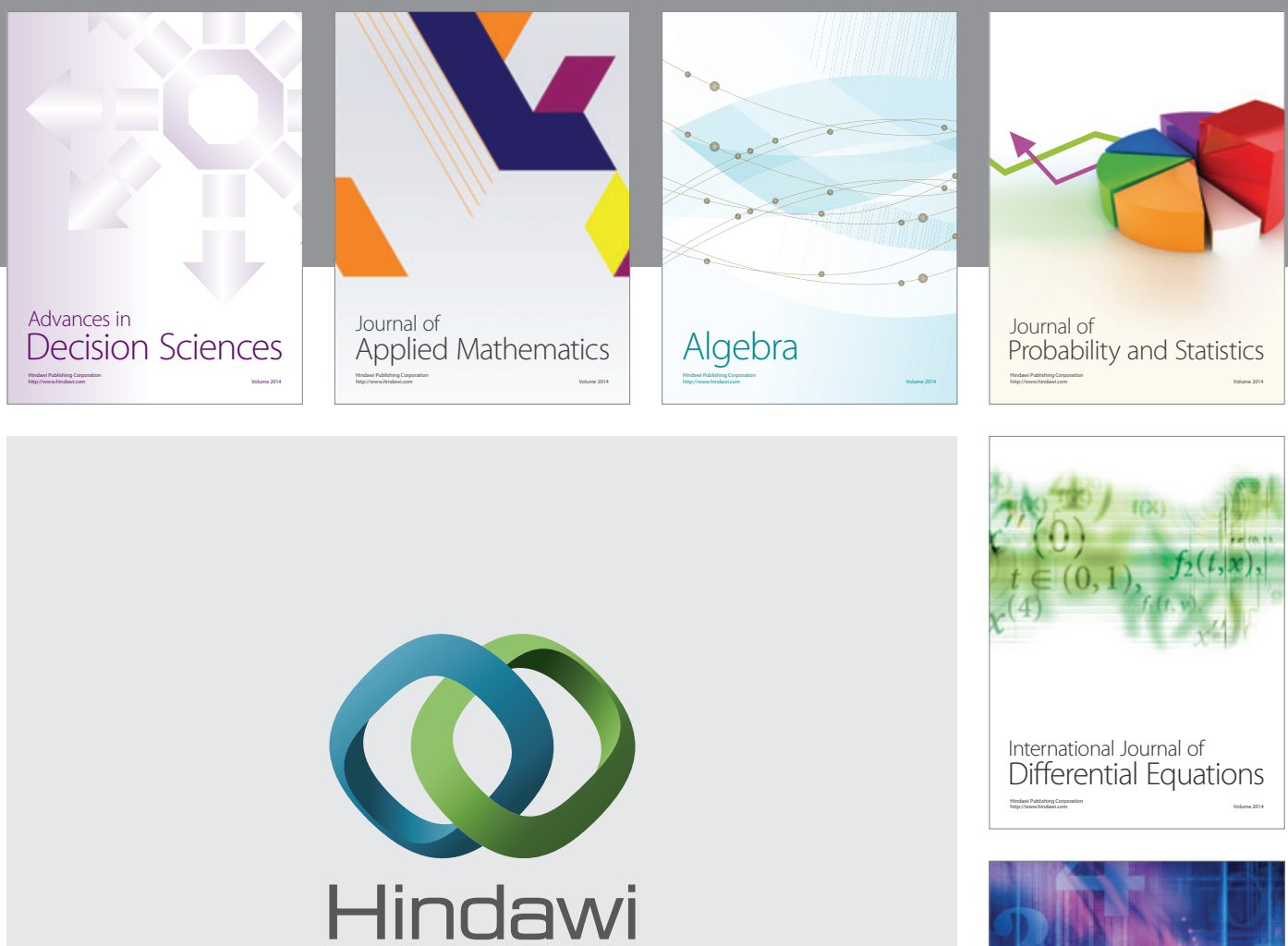

Submit your manuscripts at http://www.hindawi.com
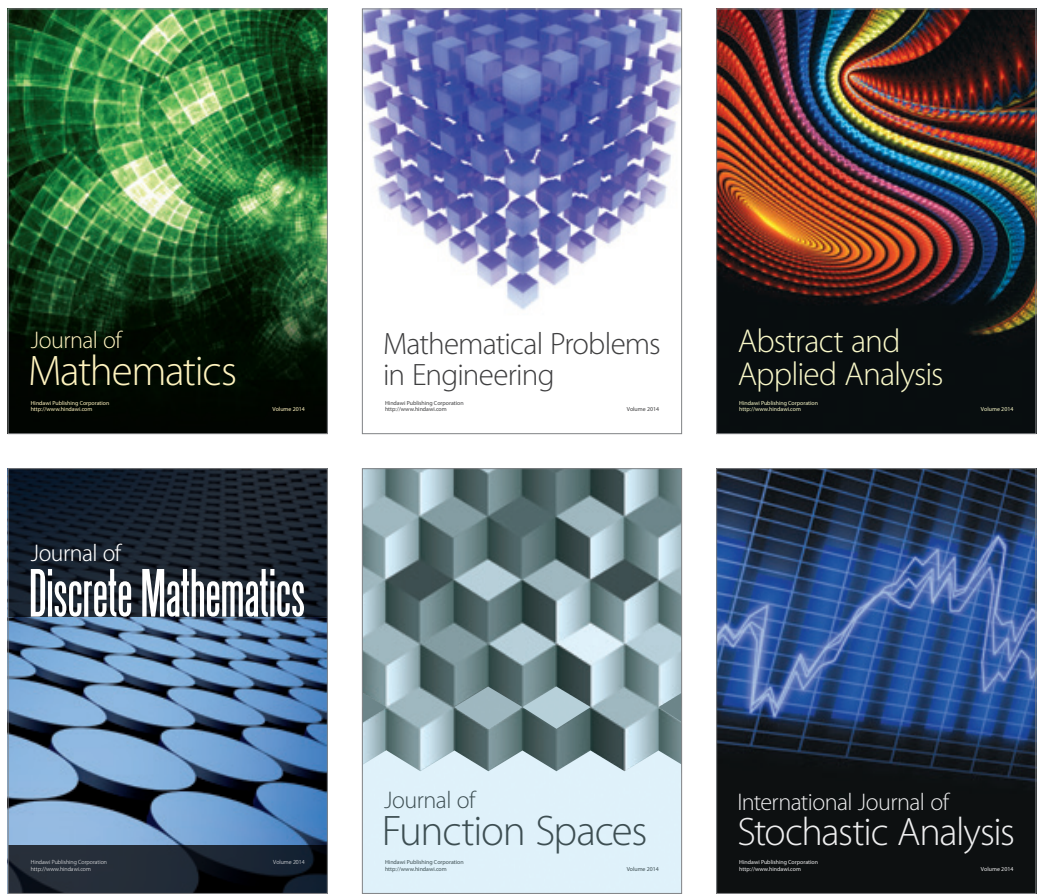

Journal of

Function Spaces

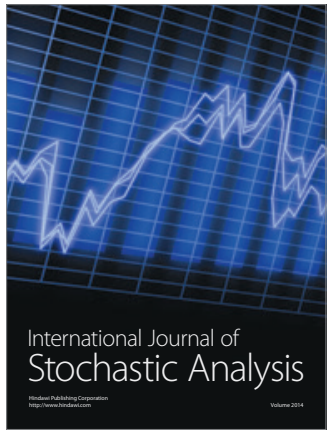

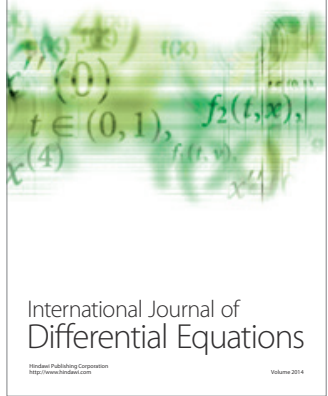
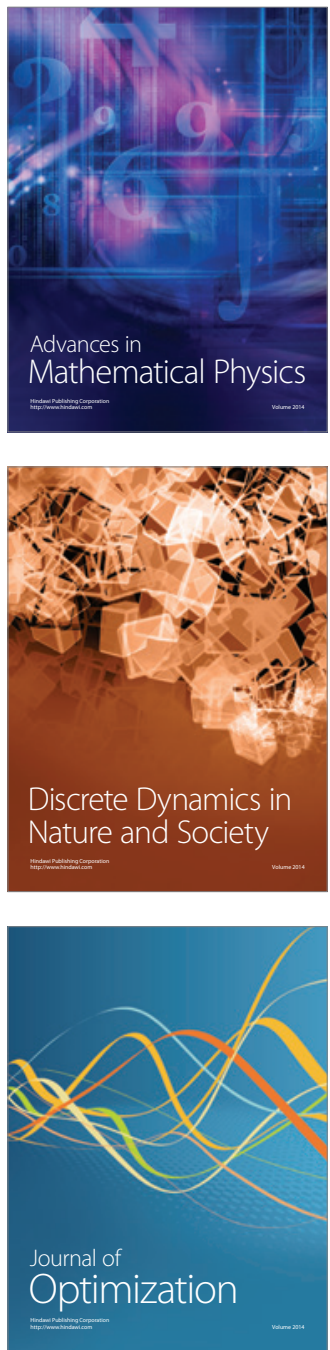